\title{
CONFORMAL MAPPING, CONVEXITY AND TOTAL ABSOLUTE CURVATURE
}

\author{
MARIA KOUROU
}

\begin{abstract}
Let $f$ be a holomorphic and locally univalent function on the unit disk $\mathbb{D}$. Let $C_{r}$ be the circle centered at the origin of radius $r$, where $0<r<$ 1. We will prove that the total absolute curvature of $f\left(C_{r}\right)$ is an increasing function of $r$. Moreover, we present inequalities involving the $\mathrm{L}^{p}$-norm of the curvature of $f\left(C_{r}\right)$. Using the hyperbolic geometry of $\mathbb{D}$, we will prove an analogous monotonicity result for the hyperbolic total curvature. In the case where $f$ is a hyperbolically convex mapping of $\mathbb{D}$ into itself, we compare the hyperbolic total curvature of the curves $C_{r}$ and $f\left(C_{r}\right)$ and show that their ratio is a decreasing function. The last result can also be seen as a geometric version of the classical Schwarz Lemma.
\end{abstract}

\section{INTRODUCTION}

Let $f$ be a conformal mapping on the unit disk $\mathbb{D}$. Let's denote the euclidean disk

$$
r \mathbb{D}=\{z \in \mathbb{C}:|z|<r\}
$$

and the circle

$$
C_{r}=\{z \in \mathbb{C}:|z|=r\}
$$

for $0<r<1$.

The curve $f\left(C_{r}\right)$ is smooth, simple, and closed for every $r \in(0,1)$. Also, $f\left(C_{r}\right)$ possesses a potential theoretic meaning, since it is a level curve of the Green function on $f(\mathbb{D})$ with pole at $f(0)$.

For sufficiently small radius $r$, the curve $f\left(C_{r}\right)$ is almost a circle. In fact, the isoperimetric ratio $\frac{\mathrm{L}^{2} f\left(C_{r}\right)}{\mathrm{A} f(r \mathbb{D})}$ approaches $4 \pi$, as $r \rightarrow 0$, where $\mathrm{L}$ and $\mathrm{A}$ denote the length and the area, respectively; see [10]. Moreover, for every $r \leq 2-\sqrt{3}$, the curve $f\left(C_{r}\right)$ is convex and $f(r \mathbb{D})$ is a convex domain; see [9, Theorem 2.13]. The number $2-\sqrt{3}$ is called radius of convexity and it is a sharp bound regarding the convexity of the domain $f(r \mathbb{D})$. The question that arises at this point is what happens when $r$ is greater than the radius of convexity. And what if the function $f$ is not univalent but only locally univalent?

A holomorphic function $f$ is convex if it is univalent and its image $f(\mathbb{D})$ is a euclidean convex domain. An equivalent definition of a convex function is the following. A holomorphic and locally univalent function $f: \mathbb{D} \rightarrow \mathbb{C}$ is a convex

Received by the editors June 29, 2017, and, in revised form, November 23, 2017, and January $25,2018$.

2010 Mathematics Subject Classification. Primary 30C45, 30C35.

Key words and phrases. Total absolute curvature, hyperbolic convexity, conformal mapping, convexity. 
univalent function if and only if the harmonic function

$$
v(z):=\operatorname{Re}\left\{1+z \frac{f^{\prime \prime}(z)}{f^{\prime}(z)}\right\}
$$

is strictly positive, for every $z \in \mathbb{D}$. Furthermore, according to a theorem of Study [27], if $f$ is a convex function, then $f(r \mathbb{D})$ is a convex domain for every $r \in(0,1)$. More information on convex univalent functions can be found in [9], 12, [17, and [23].

We need some kind of measurement to show us whether $f\left(C_{r}\right)$ is a convex curve or not and how much it diverges from being convex. The most suitable geometric quantity with this property is the total absolute curvature of $f\left(C_{r}\right)$. A beneficial factor for examining this quantity is the fact that it combines the notions of curvature and convexity. Besides that, the total absolute curvature of $f\left(C_{r}\right)$ measures how far $f\left(C_{r}\right)$ is from being a convex curve and, having a positive sign, it provides some kind of distance between a function and its convexity.

For the definition of total absolute curvature, let $\gamma$ be a smooth curve in $\mathbb{D}$. We denote by $\kappa(z, \gamma)$ the signed euclidean curvature of $\gamma$ at the point $z \in \gamma$. The total absolute curvature of $\gamma$ is the quantity

$$
\int_{\gamma}|\kappa(z, \gamma)||d z|
$$

It is known that the total absolute curvature of a smooth and closed curve is always greater than $2 \pi$, with equality holding if and only if the curve $\gamma$ is convex. This result was first proved by W. Fenchel; see [26, Corollary 6.18].

Let $f$ be a holomorphic and locally univalent function on $\mathbb{D}$. By $\kappa(w, f(\gamma))$ we denote the euclidean curvature of $f(\gamma)$ at the point $w \in f(\gamma)$. Therefore, the quantity

$$
\int_{f(\gamma)}|\kappa(w, f(\gamma))||d w|
$$

is the total absolute curvature of $f(\gamma)$ and hence, the greater this quantity becomes, the less convex the function $f$ is.

Theorem 1.1. Let $f$ be a holomorphic and locally univalent function on $\mathbb{D}$. Then

$$
r \mapsto \int_{f\left(C_{r}\right)}\left|\kappa\left(w, f\left(C_{r}\right)\right)\right||d w|
$$

is a strictly increasing log-convex function of $r \in(0,1)$, except when $f$ is convex. In this case, it is constant and equal to $2 \pi$. Moreover,

$$
\lim _{r \rightarrow 0} \int_{f\left(C_{r}\right)}\left|\kappa\left(w, f\left(C_{r}\right)\right)\right||d w|=2 \pi .
$$

At this point, we should notice that the total absolute curvature of $C_{r}$ is constant. Since $C_{r}$ is a circle, and therefore a convex curve, its total absolute curvature is equal to $2 \pi$. As a result, the function

$$
\Phi(r)=\frac{\int_{f\left(C_{r}\right)}\left|\kappa\left(w, f\left(C_{r}\right)\right)\right||d w|}{\int_{C_{r}}\left|\kappa\left(z, C_{r}\right)\right||d z|}=\frac{1}{2 \pi} \int_{f\left(C_{r}\right)}\left|\kappa\left(w, f\left(C_{r}\right)\right)\right||d w|,
$$

which is the ratio of the total absolute curvature of $f\left(C_{r}\right)$ to the total absolute curvature of $C_{r}$, is an increasing function. 
So, Theorem 1.1 is a monotonicity result regarding the total absolute curvature of $f\left(C_{r}\right)$, compared to the total absolute curvature of $C_{r}$. This reminds us of similar monotonicity results that are applications and geometric versions of the classical Schwarz Lemma. Geometric quantities are used in order to measure the size of the image $f(r \mathbb{D})$ or $f\left(C_{r}\right)$, compared to the size of $r \mathbb{D}$ or $C_{r}$, respectively. Such geometric quantities are area, length, logarithmic capacity, diameter, inner radius, etc., as we can see in [4, [5, 7], and [8].

The first result of this kind is probably the theorem of G. Pólya and G. Szegö, who used length as the geometric quantity and proved that

$$
r \mapsto \frac{\operatorname{Length}\left(f\left(C_{r}\right)\right)}{\operatorname{Length}\left(C_{r}\right)}=\frac{\int_{0}^{2 \pi}\left|f^{\prime}\left(r e^{i t}\right)\right| d t}{2 \pi}
$$

is an increasing function of $r \in(0,1)$, in $[22$. In 2 and 8 , monotonicity results for the area and logarithmic capacity of the images of a holomorphic function are proved. The functions

$$
\Phi_{A}(r)=\frac{\text { Area } f(r \mathbb{D})}{\pi r^{2}} \text { and } \Phi_{C}(r)=\frac{\operatorname{Cap} f(r \mathbb{D})}{r}
$$

are increasing for $r \in(0,1)$. Therefore, Theorem 1.1 is a similar monotonicity result regarding the total absolute curvature.

Next, we define the function

$$
\Phi_{p}(r)=\frac{\int_{f\left(C_{r}\right)}\left|\kappa\left(w, f\left(C_{r}\right)\right)\right|^{p}|d w|}{\int_{C_{r}}\left|\kappa\left(z, C_{r}\right)\right|^{p}|d z|}, \quad 0<r<1
$$

for $p \in \mathbb{R}$. When the function $f$ is not convex, the curvature of $f\left(C_{r}\right)$ is equal to zero at some point $w$. So, the function $\Phi_{p}(r)$ is not defined when $p<0$ and $f$ is not convex.

For $p=0$, the function $\Phi_{0}(r)$ has the form of (1.5) and it is an increasing function. For a convex function $f$, when $p=2$, the function $\Phi_{2}(r)$ is the ratio of elastic energy of the curve $f\left(C_{r}\right)$ to the elastic energy of $C_{r}$ (see [10] and [14]). Moreover, when $p=-1$, the integrand is the radius of curvature of each curve. For negative values of $p$, we have the following theorem.

Theorem 1.2. If $f$ is a convex univalent function in $\mathbb{D}$, the functions $\Phi_{p}(r)$ and $\log \Phi_{p}(r)$, for $p<0$, are strictly increasing log-convex functions of $r \in(0,1)$, except when $f$ is linear. In this case, $\Phi_{p}(r)$ is constant and equal to $\left|f^{\prime}(0)\right|^{1-p}$. Moreover,

$$
\lim _{r \rightarrow 0} \Phi_{p}(r)=\left|f^{\prime}(0)\right|^{1-p} .
$$

We should also remark that there are not any monotonicity results for the function $\Phi_{p}(r)$ for the rest of the cases on $f$ and $p$; we present some counterexamples in Section 4. The following theorem contains inequalities involving the $\mathrm{L}^{p}$-norm of the curvature of $f\left(C_{r}\right)$.

Theorem 1.3. If $f$ is a holomorphic and locally univalent function on $\mathbb{D}$, then for $p>1$,

$$
\int_{f\left(C_{r}\right)}\left|\kappa\left(w, f\left(C_{r}\right)\right)\right|^{p}|d w| \geq 2 \pi r^{1-p}
$$


whereas, for $0<p<1$, the following inequality holds:

$$
\int_{f\left(C_{r}\right)}\left|\kappa\left(w, f\left(C_{r}\right)\right)\right|^{p}|d w|>2 \pi r^{1-p}\left|f^{\prime}(0)\right|^{p} .
$$

Equality in (1.8) occurs only when $f$ is a convex function and $p=1$.

The results so far concern the euclidean case, where $f(\mathbb{D}) \subset \mathbb{C}$. In Sections 6 and 7. we examine whether there are any similar monotonicity results when $\mathbb{D}$ is seen from a hyperbolic perspective.

We recall that $\lambda_{\mathbb{D}}$ is the density of the hyperbolic metric in $\mathbb{D}$ and that the hyperbolic metric

$$
\lambda_{\mathbb{D}}(z)|d z|=\frac{|d z|}{1-|z|^{2}}:=d s
$$

has constant Gaussian curvature equal to -4 .

The hyperbolic distance in the unit disk, for $a, b \in \mathbb{D}$ is equal to

$$
d_{\mathbb{D}}(a, b)=\operatorname{arctanh}\left|\frac{a-b}{1-\bar{a} b}\right|
$$

and it is invariant under the group of conformal self-maps of $\mathbb{D}$. For more information on the hyperbolic metric and, in general, on the hyperbolic geometry of the unit disk, the reader can refer to [3] and [16].

We recall that a subregion $\Omega$ of the unit disk is hyperbolically convex if for every pair of points in $\Omega$ the hyperbolic geodesic arc in $\mathbb{D}$ that joins them, lies in $\Omega$; see [16. The hyperbolic geodesic curves of $\mathbb{D}$ are the arcs of euclidean circles that are orthogonal to the boundary.

From [16], a conformal map $f: \mathbb{D} \rightarrow \mathbb{D}$ is called hyperbolically convex if $f(\mathbb{D})$ is a hyperbolically convex subregion of $\mathbb{D}$. An equivalent definition is that the function $f$, which is a holomorphic and locally univalent function in $\mathbb{D}$ with $f(\mathbb{D}) \subset \mathbb{D}$, is hyperbolically convex if and only if

$$
u(z):=\operatorname{Re}\left\{1+\frac{z f^{\prime \prime}(z)}{f^{\prime}(z)}+\frac{2 z f^{\prime}(z) \overline{f(z)}}{1-|f(z)|^{2}}\right\}>0
$$

for every $z \in \mathbb{D}$. From $[20$, the above function is subharmonic on $\mathbb{D}$ if and only if $f$ is hyperbolically convex.

Using the hyperbolic analogue of Study's Theorem, which is proved in [16, we have an equivalent definition for hyperbolically convex functions. Suppose $f$ is a conformal map with $f(\mathbb{D}) \subset \mathbb{D}$. Then $f$ is hyperbolically convex if and only if $f$ maps every subdisk of $\mathbb{D}$ onto a hyperbolically convex region. In particular, for $0<r<1$, the function $f(r z)$ is hyperbolically convex. Further information on hyperbolic convexity can be seen in [15], 16], and [18].

The hyperbolic disk centered at the origin of radius $\rho$

$$
D_{h}(0, \rho)=\{z \in \mathbb{D}: \operatorname{arctanh}|z|<\rho\}=\{z \in \mathbb{D}:|z|<\tanh \rho\}
$$

is a euclidean disk centered at the origin of radius $r:=\tanh \rho$. Its boundary is

$$
\partial D_{h}(0, \rho)=\{z \in \mathbb{D}:|z|=\tanh \rho\}=\partial D(0, r)=C_{r},
$$

where $r=\tanh \rho$.

Furthermore, when $f$ is a conformal mapping of $\mathbb{D}$ into itself, the curve $f\left(C_{r}\right)$ is hyperbolically convex for $r \leq 2-\sqrt{3}$; see [16]. The number $2-\sqrt{3}$ is also called radius of hyperbolic convexity and it is a sharp bound regarding the hyperbolic 
convexity of the domain $f(r \mathbb{D})$. As in the euclidean case, the question is what happens when $r$ is greater than $2-\sqrt{3}$ or when $f$ is only locally univalent. In order to investigate the hyperbolic analogue of Theorem 1.1, we use the hyperbolic curvature of the curve $f\left(C_{r}\right)$.

Let $\gamma$ be a smooth curve in the unit disk $\mathbb{D}$, with non-vanishing derivative, and let $f$ be a holomorphic and locally univalent map with $f(\mathbb{D}) \subset \mathbb{D}$. The hyperbolic curvature of $\gamma$ at the point $z \in \gamma$ is denoted by $\kappa_{h}(z, \gamma)$, whereas, the hyperbolic curvature of $f \circ \gamma$ at a point $f(z), z \in \gamma$, is denoted by $\kappa_{h}(f(z), f \circ \gamma)$.

We should note that the hyperbolic curvature on the unit disk is invariant under conformal self-maps of $\mathbb{D}$. For the total hyperbolic curvature of the curve $f\left(C_{r}\right)$ in the unit disk, we will prove the following monotonicity result.

Theorem 1.4. Let $f$ be a holomorphic and locally univalent function on $\mathbb{D}$ with $f(\mathbb{D}) \subset \mathbb{D}$. Then

$$
r \mapsto \int_{f\left(C_{r}\right)} \kappa_{h}\left(w, f\left(C_{r}\right)\right) \lambda_{\mathbb{D}}(w)|d w|, \quad 0<r<1
$$

is a strictly increasing function.

Similar monotonicity results have been proved in 6 . The functions

$$
r \mapsto \frac{\mathrm{R}_{\mathrm{h}} f(r \mathbb{D})}{r} \quad \text { and } \quad r \mapsto \frac{\operatorname{caph} f(r \mathbb{D})}{r}
$$

are increasing functions of $r \in(0,1)$, where $R_{h}$ is the hyperbolic-area-radius of $f(r \mathbb{D})$ and caph denotes the hyperbolic capacity. We will prove a monotonicity result for the function which is the hyperbolic analogue of $\Phi(r)$.

We define the function

$$
\Phi_{h}(r)=\frac{\int_{f\left(C_{r}\right)}\left|\kappa_{h}\left(w, f\left(C_{r}\right)\right)\right| d s}{\int_{C_{r}}\left|\kappa_{h}\left(z, C_{r}\right)\right| d s}, \quad 0<r<1,
$$

which is the ratio of the hyperbolic total absolute curvature of $f\left(C_{r}\right)$ to the hyperbolic total absolute curvature of $C_{r}$.

Theorem 1.5. Let $f$ be a hyperbolically convex function in $\mathbb{D}$, with $f(\mathbb{D}) \subset \mathbb{D}$. Then $\Phi_{h}(r)$ is a strictly decreasing function of $r \in(0,1)$, except when $f$ is a conformal self-map of the unit disk. In that case, $\Phi_{h}$ is constant and equal to 1.

From Theorem 1.5, we obtain the following Schwarz-type result.

Corollary 1.1. Suppose $f$ is a hyperbolically convex function in $\mathbb{D}$. Then

$$
\int_{f\left(C_{r}\right)} \kappa_{h}\left(w, f\left(C_{r}\right)\right) d s \leq \int_{C_{r}} \kappa_{h}\left(z, C_{r}\right) d s=\frac{2 \pi\left(1+r^{2}\right)}{1-r^{2}}
$$

for every $r \in(0,1)$, with equality holding if and only if $f$ is a conformal self-map of $\mathbb{D}$.

In the proofs of Theorems 1.4 and 1.5, we will use the Gauss-Bonnet formula in the following form; see [26, Theorem 6.5]. Let $M$ be an oriented two-dimensional Riemannian manifold, with Gaussian curvature $K$ and volume element $d A$. Let $N \subset M$ be a compact two-dimensional manifold-with-boundary which is diffeomorphic to a subset of $\mathbb{R}^{2}$ and whose boundary is connected. Let $d s$ be the volume 
element of $\partial N$ and let $\kappa$ be the signed geodesic curvature of $\partial N$. Then

$$
\int_{N} K d A+\int_{\partial N} \kappa d s=2 \pi
$$

The unit disk $\mathbb{D}$ endowed with the hyperbolic metric is a two-dimensional Riemannian manifold of constant Gaussian curvature equal to -4 . If $\Omega$ is a hyperbolic domain in $\mathbb{D}$, the Gauss-Bonnet formula (1.13) has the form

$$
-4 \mathrm{~A}_{\mathrm{h}}(\Omega)+\int_{\gamma} \kappa_{h}(z, \gamma) \lambda_{\mathbb{D}}(z)|d z|=2 \pi,
$$

where $\gamma$ is the boundary of $\Omega$ and it is a smooth, simple, and closed curve in $\mathbb{D}$.

The article is structured in the following way. In Section 2, we calculate the total absolute curvature of $f\left(C_{r}\right)$ and prove that it is an increasing function, as stated in Theorem 1.1. In Section 3, we study the case where $f$ is a convex function and prove Theorem 1.2, whereas, in Section 4, counterexamples on the monotonicity of $\Phi_{p}(r)$ are presented, depending on the values of $p$ and the convexity of the function $f$. In Section 5, we prove the inequalities of Theorem 1.3. Thereafter, the unit disk is explored from a hyperbolic point of view. More specifically, in Section 6 . we prove that the hyperbolic total curvature is an increasing function, as stated in Theorem 1.4 and we examine some results concerning the function $u$, whose sign indicates the hyperbolic convexity of the function $f$. In Section 7 information on the monotonicity of the function $\Phi_{h}$ is extracted and Theorem 1.5] and Corollary 1.1 are proved.

\section{Proof of Theorem 1.1}

The complex plane $\mathbb{C}$ endowed with the euclidean metric is a two-dimensional Riemannian manifold. The Gaussian curvature of the euclidean metric is equal to zero. As a result, if $\Omega$ is a domain in $\mathbb{C}$, from (1.13), it follows that

$$
\int_{\gamma} \kappa(z, \gamma)|d z|=2 \pi
$$

where $\gamma$ is the boundary of $\Omega$ and it is a smooth, simple, and closed curve.

To begin with, we will calculate the euclidean total absolute curvature of $f\left(C_{r}\right)$. If $f$ is a holomorphic and locally univalent function on $\mathbb{D}$, the euclidean curvature of $f\left(C_{r}\right)$ is given by the formula

$$
\kappa\left(f(z), f\left(C_{r}\right)\right)=\frac{1}{|z|\left|f^{\prime}(z)\right|} v(z),
$$

where the function $v$ is defined in (1.1) and it is harmonic on $\mathbb{D}$ as the real part of a holomorphic function; see [19]. Consequently, the total absolute curvature of $f\left(C_{r}\right)$ is given by the formula

$$
\begin{aligned}
\int_{f\left(C_{r}\right)}\left|\kappa\left(w, f\left(C_{r}\right)\right)\right||d w| & =\int_{C_{r}}\left|\kappa\left(f(z), f\left(C_{r}\right)\right)\right|\left|f^{\prime}(z)\right||d z| \\
& =\int_{C_{r}} \frac{1}{r\left|f^{\prime}(z)\right|}|v(z)|\left|f^{\prime}(z)\right||d z| \\
& =\int_{0}^{2 \pi} \frac{1}{r}\left|v\left(r e^{i t}\right)\right| r d t=\int_{0}^{2 \pi}\left|v\left(r e^{i t}\right)\right| d t .
\end{aligned}
$$


In order to acquire results on the strict monotonicity mentioned in Theorem 1.1 . we will need the following helpful lemmas. For a continuous function $u$ on $C_{r}$, let's define its integral mean as

$$
m(r, u)=\frac{1}{2 \pi} \int_{0}^{2 \pi} u\left(r e^{i t}\right) d t .
$$

Lemma 2.1. Suppose that $u$ is continuous and subharmonic in the disk $\{z \in \mathbb{D}$ : $|z|<R\}$. Then for $0 \leq r_{1}<r_{2}<R$, either $m\left(r_{1}, u\right)<m\left(r_{2}, u\right)$, or $u$ is harmonic in $\left\{z \in \mathbb{D}:|z|<r_{2}\right\}$ and $m(r, u)$ is constant on $\left[0, r_{2}\right]$.

Proof. Let $h$ be the unique function that is continuous in $\left\{z \in \mathbb{D}:|z| \leq r_{2}\right\}$, harmonic in the disk $\left\{z \in \mathbb{D}:|z|<r_{2}\right\}$, and equal to $u$ on $C_{r_{2}}$. Then it holds either that $u<h$ or that $u=h$ in $\left\{z \in \mathbb{D}:|z|<r_{2}\right\}$. Let's also notice that since $h$ is harmonic, the function $m(r, h)$ is constant and equal to $h(0)$ for $r \in\left[0, r_{2}\right)$. Hence, we obtain that either

$$
m\left(r_{1}, u\right)<m\left(r_{1}, h\right)=m\left(r_{2}, h\right)=m\left(r_{2}, u\right),
$$

or $u$ is harmonic in the disk $\left\{z \in \mathbb{D}:|z|<r_{2}\right\}$, in which case its integral mean $m(r, u)$ is constant for $r \in\left[0, r_{2}\right]$.

Lemma 2.2 ([13, Exercise 4, p. 80]). Suppose that $u$ is a continuous subharmonic function in the annulus $\left\{z \in \mathbb{D}: R_{1}<|z|<R_{2}\right\}$. Then for $R_{1}<r_{1}<r<r_{2}<R_{2}$,

$$
m(r, u) \leq \frac{\log r_{2}-\log r}{\log r_{2}-\log r_{1}} m\left(r_{1}, u\right)+\frac{\log r-\log r_{1}}{\log r_{2}-\log r_{1}} m\left(r_{2}, u\right) .
$$

Equality holds if and only if $u$ is harmonic in $\left\{z \in \mathbb{D}: r_{1}<|z|<r_{2}\right\}$.

The inequality (2.4) states that the integral mean of a subharmonic function satisfies Hadamard's Theorem and, consequently, $m(r, u)$ is log-convex in the annulus $\left\{z \in \mathbb{D}: R_{1}<|z|<R_{2}\right\}$.

Completion of the Proof of Theorem 1.1. As we observe in (2.3), the total absolute curvature of $f\left(C_{r}\right)$ is the integral mean of the function $|v|$, which is subharmonic on $\mathbb{D}$, since

$$
v(z)=\operatorname{Re}\left\{1+z \frac{f^{\prime \prime}(z)}{f^{\prime}(z)}\right\}
$$

is harmonic on $\mathbb{D}$. Hence, according to [25, Theorem 2.6.8], the total absolute curvature of $f\left(C_{r}\right)$ is an increasing log-convex function of $r \in(0,1)$.

In the Introduction, we have defined the function $\Phi(r)$ in (1.4), which can also be written as

$$
\Phi(r)=\frac{1}{2 \pi} \int_{0}^{2 \pi}\left|v\left(r e^{i t}\right)\right| d t=m(r,|v|) .
$$

The monotonicity of the function $\Phi(r)$ coincides with the monotonicity of the total absolute curvature of $f\left(C_{r}\right)$. So, for the proof of Theorem 1.1, it suffices to prove that $\Phi(r)$ is a strictly increasing log-convex function, unless $f$ is convex.

The function $|v|$ is continuous and subharmonic on $\mathbb{D}$, thus we can apply Lemma 2.1 and conclude that either

$$
\Phi\left(r_{1}\right)=m\left(r_{1},|v|\right)<m\left(r_{2},|v|\right)=\Phi\left(r_{2}\right),
$$

for $0 \leq r_{1}<r_{2}<1$, or $|v|$ is harmonic on $\mathbb{D}$. So, it follows that $\Phi(r)$ is either strictly increasing or constant in $[0,1)$. 
Moreover, we can apply Lemma 2.2 and obtain that $\Phi(r)$ is strictly log-convex in the annulus $\left\{z \in \mathbb{D}: R_{1}<|z|<R_{2}\right\}$, unless $|v|$ is harmonic on $\left\{z \in \mathbb{D}: R_{1}<|z|<\right.$ $\left.R_{2}\right\}$. The choice of $R_{1}$ and $R_{2}$ was arbitrary, so we can take the limits $R_{1} \rightarrow 0$ and $R_{2} \rightarrow 1$. Hence, $\Phi(r)$ is a strictly log-convex function of $r \in(0,1)$, unless $|v|$ is a harmonic function on $\mathbb{D}$.

If $|v|$ is harmonic on $\mathbb{D}$, since $v$ is also harmonic, it must hold that either $v$ preserves its sign, for every $z \in \mathbb{D}$, or $v=0$ everywhere in the unit disk. However, $v(0)=1$ that leads to $v$ being positive, for every $z \in \mathbb{D}$. This means that $f$ is a convex univalent function in the unit disk and

$$
\Phi(r)=\lim _{r \rightarrow 0} \Phi(r)=\lim _{r \rightarrow 0} m(r,|v|)=v(0)=1 .
$$

Therefore, it was proved that the function $\Phi(r)$ is a strictly increasing log-convex function of $r \in(0,1)$, unless $f$ is a convex univalent function.

For the reverse claim, if $f$ is convex, then $v(z)>0$, for every $z \in \mathbb{D}$, and so $\Phi(r)$ is constant and equal to $v(0)=1$, for every $r \in(0,1)$.

As a result, the function in (1.2) is a strictly increasing log-convex function of $r \in(0,1)$, unless $f$ is a convex univalent function. If $f$ is convex, it is equal to $2 \pi$. The value of the limit in (1.3) follows directly from (2.5).

\section{Proof of Theorem 1.2}

Let $p \in \mathbb{R}$. We have defined the function $\Phi_{p}(r)$ in (1.6), which according to the calculations in Section 2, can be written as

$$
\begin{aligned}
\Phi_{p}(r) & =\frac{1}{\int_{0}^{2 \pi} r^{1-p} d t} \int_{C_{r}}\left|\kappa\left(f(z), f\left(C_{r}\right)\right)\right|^{p}\left|f^{\prime}(z)\right||d z| \\
& =\frac{r^{p-1}}{2 \pi} \int_{C_{r}} \frac{1}{|z|^{p}\left|f^{\prime}(z)\right|^{p}}|v(z)|^{p}\left|f^{\prime}(z)\right||d z| \\
& =\frac{r^{p-1}}{2 \pi} \int_{0}^{2 \pi} r^{1-p}\left|v\left(r e^{i t}\right)\right|^{p}\left|f^{\prime}\left(r e^{i t}\right)\right|^{1-p} d t=\frac{1}{2 \pi} \int_{0}^{2 \pi}\left|v\left(r e^{i t}\right)\right|^{p}\left|f^{\prime}\left(r e^{i t}\right)\right|^{1-p} d t .
\end{aligned}
$$

According to Theorem 1.2, we assume that $f$ is a convex function and $p<0$. Let's define the function $h(z)=v^{p}(z)\left|f^{\prime}(z)\right|^{1-p}$, for $z \in \mathbb{D}$. So, $\Phi_{p}(r)$ is the integral mean of the function $h$. The logarithm of $h$ can be written as

$$
\log h(z)=p \log v(z)+(1-p) \log \left|f^{\prime}(z)\right| .
$$

From [25. Exercise 2, p. 47], since $-\log x$ is a convex function of $x \in(0,1)$, we obtain that $-\log v(z)$ is subharmonic on the unit disk $\mathbb{D}$ and so, the function $\log v(z)$ is superharmonic on $\mathbb{D}$.

As a result, $\log h(z)$ is subharmonic on $\mathbb{D}$, as the sum of subharmonic and harmonic functions in (3.1). Therefore, $h(z)$ belongs in the class PL of subharmonic functions. This means that the function itself is subharmonic and its integral mean $\Phi_{p}(r)$ is an increasing log-convex function of $r \in(0,1)$. Moreover, $\log \Phi_{p}(r)$ is increasing and by [24, $\S 2.16]$, it is also log-convex.

Using Lemma 2.1 from Section 2, we obtain that $\Phi_{p}(r)$ and $\log \Phi_{p}(r)$ are strictly increasing functions of $r \in(0,1)$, unless $h$ is harmonic on $\mathbb{D}$. Moreover, using Lemma 2.2 and assuming that $R_{1} \rightarrow 0$ and $R_{2} \rightarrow 1$, since their choice was arbitrary, the functions $\Phi_{p}(r)$ and $\log \Phi_{p}(r)$ are also strictly log-convex in $(0,1)$, unless $h$ is harmonic on $\mathbb{D}$. 
In the case where $h$ is a harmonic function on $\mathbb{D}, \Phi_{p}(r)$ is constant and equal to $h(0)=v^{p}(0)\left|f^{\prime}(0)\right|^{1-p}=\left|f^{\prime}(0)\right|^{1-p}$. In addition, $\log h$ is a superharmonic function on $\mathbb{D}$, since it is the logarithm of a harmonic function, as stated above, from [25, Exercise 2, p. 47].

However, from (3.1), the function $\log h$ is both subharmonic and superharmonic; hence it is harmonic on $\mathbb{D}$. If we denote by $\Delta$ the Laplacian of a $\mathcal{C}^{2}$-function, it holds that

$$
\Delta \log h=-\frac{1}{h^{2}}\left[\left(\frac{\partial h}{\partial x}\right)^{2}+\left(\frac{\partial h}{\partial y}\right)^{2}\right]+\frac{1}{h} \Delta h,
$$

and since the functions $h$ and $\log h$ are harmonic on $\mathbb{D}$, we have that

$$
\left(\frac{\partial h}{\partial x}\right)^{2}+\left(\frac{\partial h}{\partial y}\right)^{2}=0
$$

for $z=x+i y \in \mathbb{D}$. As a result, the function $h$ is constant on $\mathbb{D}$, equal to $\left|f^{\prime}(0)\right|^{1-p}$ and it is true that

$$
v^{p}(z)\left|f^{\prime}(z)\right|^{1-p}=\left|f^{\prime}(0)\right|^{1-p}
$$

for every $z \in \mathbb{D}$. Therefore,

$$
p \log v(z)=(1-p) \log \left|f^{\prime}(0)\right|-(1-p) \log \left|f^{\prime}(z)\right|
$$

and it follows that $\log v$ is harmonic on $\mathbb{D}$, since $\log \left|f^{\prime}\right|$ is harmonic for a locally injective function $f$. Writing the equation (3.2) for $v$ and considering the fact that $v$ and $\log v$ are both harmonic functions on $\mathbb{D}$, we attain that $v$ is constant on $\mathbb{D}$ and equal to $v(0)=1$. This implies that $f^{\prime \prime}(z)=0$, for every $z \in \mathbb{D}$, which happens if and only if $f^{\prime}$ is constant, or subsequently, when $f$ is linear.

For the reverse claim, we assume that $f$ is linear. Then $v(z)=1$ and $\left|f^{\prime}(z)\right|=$ $\left|f^{\prime}(0)\right|$, for every $z \in \mathbb{D}$. Thus,

$$
\Phi_{p}(r)=\frac{1}{2 \pi} \int_{0}^{2 \pi}\left|f^{\prime}(0)\right|^{1-p} d t=\left|f^{\prime}(0)\right|^{1-p}
$$

is constant on $(0,1)$.

Hence, $\Phi_{p}$ is constant and equal to $\left|f^{\prime}(0)\right|^{1-p}$ if and only if $f$ is linear. Moreover, the limit

$$
\lim _{r \rightarrow 0} \Phi_{p}(r)=\lim _{r \rightarrow 0} m(r, h)=\left|f^{\prime}(0)\right|^{1-p} .
$$

\section{Counterexamples}

In the previous sections, it was shown that $\Phi_{p}(r)$ is an increasing and log-convex function for $p=1$. We are going to examine what happens when $p$ takes values in the intervals $(0,1)$ and $(1,+\infty)$. As we will see by stating some counterexamples, for these values of $p$, there are not any monotonicity results for the function $\Phi_{p}$. Examining the case where the function is not convex, we consider as an example the Koebe function

$$
k(z)=\frac{z}{(1-z)^{2}},
$$

which maps the unit disk conformally onto $\mathbb{C} \backslash\left(-\infty,-\frac{1}{4}\right]$. By calculations, we have

$$
\Phi_{p}(r)=\frac{1}{2 \pi} \int_{0}^{2 \pi} \frac{\left|1-r^{4}+4 r \cos t-4 r^{3} \cos t\right|^{p}}{\left(1+r^{4}-2 r^{2} \cos (2 t)\right)^{p}} \frac{\left(1+r^{2}+2 r \cos t\right)^{\frac{1-p}{2}}}{\left(1+r^{2}-2 r \cos t\right)^{\frac{3(1-p)}{2}}} d t .
$$


Figures 1 and 2 show the graphs of the function $\Phi_{p}(r)$ for chosen values of $p$. The horizontal axis represents the values of $r$ and the vertical axis represents the values of the function $\Phi_{p}(r)$.

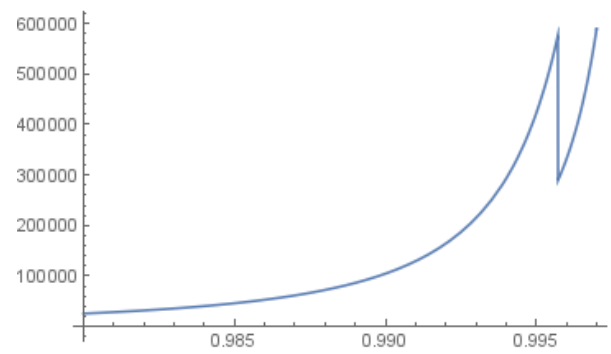

FiguRE 1. $p=2$

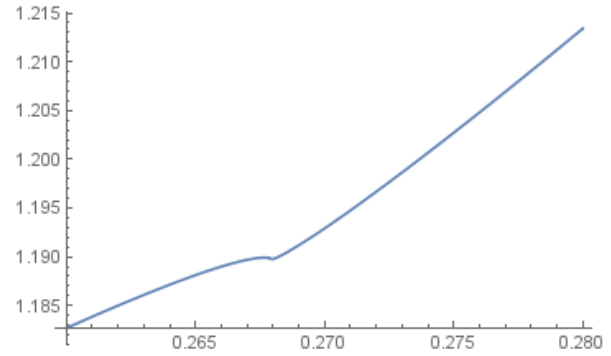

Figure 2. $p=\frac{1}{3}$

As a result, $\Phi_{p}$ is not monotonic, in general, when $p \neq 0,1$, for non-convex functions.

Examining the case where the function is convex, we have already proved that $\Phi_{p}$ is increasing as a function of $r$, for $p \leq 0$ and $p=1$. We will state a counterexample for $p \in(0,1) \cup(1,+\infty)$. Let's define the holomorphic function

$$
\sigma(z)=\sqrt{z-1}=e^{\frac{1}{2} \log (z-1)} .
$$

Then

$$
v(z)=\operatorname{Re}\left\{1+z \frac{\sigma^{\prime \prime}(z)}{\sigma^{\prime}(z)}\right\}=\frac{2+r^{2}-3 r \cos t}{2\left(1+r^{2}-2 r \cos t\right)}
$$

for $|z|=r \in(0,1)$. The quantity

$$
|1-z|^{2}=1+r^{2}-2 r \cos t>0
$$

and $1-r \cos t>0$, hence their sum

$$
2+r^{2}-3 r \cos t>0
$$

and so, $\sigma$ is a convex univalent function in $\mathbb{D}$. With calculations, it follows that

$$
\Phi_{p}(r)=\frac{1}{4 \pi} \int_{0}^{2 \pi} \frac{\left(2+r^{2}-3 r \cos t\right)^{p}}{\left(1+r^{2}-2 r \cos t\right)^{\frac{1+3 p}{4}}} d t
$$

and we can see its graphs in Figures 3 and 4 for chosen valuse of $p$.

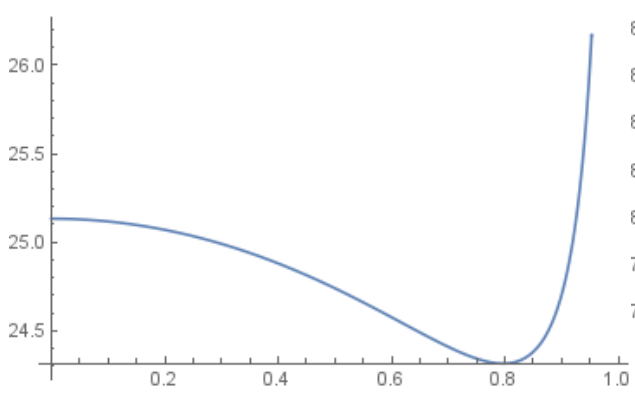

Figure 3. $p=2$

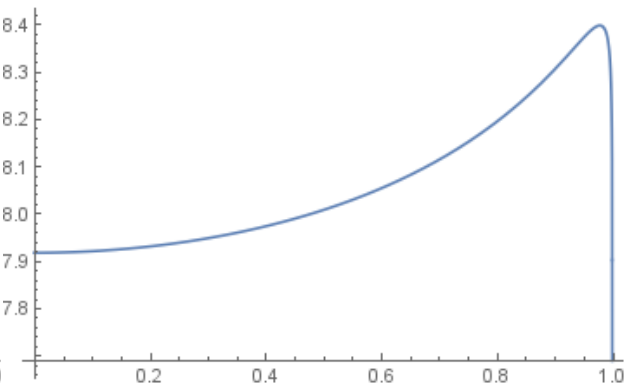

FiguRE $4 . p=\frac{1}{3}$ 
Therefore, using the above counterexamples, we can conclude that there are not any monotonicity results induced for the cases that are not included in Theorems 1.1 and 1.2 .

\section{Inequalities - Proof of Theorem 1.3}

In this section, we present lower bounds for the $\mathrm{L}^{p}$-norm of the curvature of $f\left(C_{r}\right)$, as stated in Theorem 1.3. For this purpose, the following lemma from [11, Chapter 6] is necessary.

Lemma 5.1. Let $g$ be a real function on the measure space $(X, \mu)$. The integral mean

$$
\mathcal{M}_{p}(g)=\left(\int_{X}|g|^{p} d \mu\right)^{\frac{1}{p}}
$$

is a strictly increasing function of $p, p \in \mathbb{R}$, unless $g$ is constant.

The function

$$
\Phi_{p}(r)=\frac{1}{2 \pi} \int_{0}^{2 \pi}\left(\left|v\left(r e^{i t}\right) \| f^{\prime}\left(r e^{i t}\right)\right|^{\frac{1-p}{p}}\right)^{p} d t
$$

is handled as a function of $p$ and it is the $\mathrm{L}^{p}$-norm of the function $\left|v \| f^{\prime}\right|^{\frac{1-p}{p}}$, to the power of $p$. Using its definition in (1.6), $\Phi_{p}(r)$ can also be written as

$$
\Phi_{p}(r)=\frac{r^{p-1}}{2 \pi} \int_{f\left(C_{r}\right)}\left|\kappa\left(w, f\left(C_{r}\right)\right)\right|^{p}|d w| .
$$

According to Lemma 5.1 the function $\Phi_{p}^{1 / p}(r)$ is an increasing function of $p$, for $p \in \mathbb{R}$, unless $|v|\left|f^{\prime}\right|^{\frac{1-p}{p}}$ is constant. Suppose that $|v|\left|f^{\prime}\right|^{\frac{1-p}{p}}$ is non-constant.

As a result, for $p>1, \Phi_{p}^{1 / p}(r)>\Phi_{1}(r)$ and we obtain

$$
\Phi_{p}(r)>\Phi_{1}^{p}(r)=\left(\frac{1}{2 \pi} \int_{0}^{2 \pi}\left|v\left(r e^{i t}\right)\right| d t\right)^{p} \geq 1 .
$$

Hence,

$$
\int_{f\left(C_{r}\right)}\left|\kappa\left(w, f\left(C_{r}\right)\right)\right|^{p}|d w| \geq 2 \pi r^{1-p}
$$

When $0<p<1$, it holds that

$$
\Phi_{p}^{1 / p}(r)>\Phi_{0}(r) \Leftrightarrow \Phi_{p}(r)>\Phi_{0}^{p}(r)
$$

and, therefore,

$$
\frac{r^{p-1}}{2 \pi} \int_{f\left(C_{r}\right)}\left|\kappa\left(w, f\left(C_{r}\right)\right)\right|^{p}|d w|>\left(\frac{1}{2 \pi} \int_{0}^{2 \pi}\left|f^{\prime}\left(r e^{i t}\right)\right| d t\right)^{p} .
$$

Since $\left|f^{\prime}\right|$ is subharmonic, we obtain the following inequality:

$$
\int_{f\left(C_{r}\right)}\left|\kappa\left(w, f\left(C_{r}\right)\right)\right|^{p}|d w|>2 \pi r^{1-p}\left|f^{\prime}(0)\right|^{p} .
$$

Equality at (5.2) holds if and only if we have equality in (5.1). That implies that $p=1$. Also, according to Lemma 5.1, the function $|v|\left|f^{\prime}\right|^{\frac{1-p}{p}}$ is constant and since $p=1,|v(z)|=c \geq 0$, for every $z \in \mathbb{D}$. If the constant $c$ is equal to zero, then $v(z)=0$, for every $z \in \mathbb{D}$, which means that the curvature $\kappa\left(w, f\left(C_{r}\right)\right)=0$. 
That leads to $f\left(C_{r}\right)$ being a straight line (euclidean geodesic), for every $r \in(0,1)$. However, $f\left(C_{r}\right)$ is a closed curve and so the constant $c$ cannot be equal to zero.

Thus $v(z)= \pm c$ and it preserves its sign, due to continuity. If $v$ is a negative function, then $f$ is a meromorphic concave function with a single pole at the origin, as stated in [21]. Meromorphic concave functions, though, are excluded from the class of functions that we study. Therefore, $v(z)$ is a positive function on $\mathbb{D}$ and the function $f$ is convex. As a result, equality holds at the case where $f$ is convex and $p=1$.

\section{Hyperbolic total Curvature - Proof of Theorem 1.4}

From this point on, we are interested in the case where $f$ is a holomorphic and locally univalent function on the unit disk $\mathbb{D}$ and its image $f(\mathbb{D})$ lies in $\mathbb{D}$. Here, in order to refer to the notion of curvature, we use the hyperbolic geometry of the unit disk. As stated in the Introduction, $\mathbb{D}$ is endowed with the hyperbolic metric

$$
\lambda_{\mathbb{D}}(z)|d z|=\frac{1}{1-|z|^{2}}|d z|,
$$

which has constant Gaussian curvature equal to -4 .

It is also stated in the Introduction that the hyperbolic disk $D_{h}(0, \rho), 0<\rho<1$, can be seen as a euclidean disk $r \mathbb{D}$ and its boundary is equal to $C_{r}$, where $r=\tanh \rho$. So, we are interested in calculating the geodesic curvature with respect to hyperbolic geometry of the curves $C_{r}$ and $f\left(C_{r}\right)$. For the curve $f\left(C_{r}\right)$, the following differential operators are needed:

$$
D_{h 1} f(z)=\frac{\left(1-|z|^{2}\right) f^{\prime}(z)}{1-|f(z)|^{2}}
$$

and

$$
D_{h 2} f(z)=\frac{\left(1-|z|^{2}\right)^{2} f^{\prime \prime}(z)}{1-|f(z)|^{2}}+\frac{2\left(1-|z|^{2}\right)^{2} f^{\prime}(z)^{2} \overline{f(z)}}{\left(1-|f(z)|^{2}\right)^{2}}-\frac{2 \bar{z}\left(1-|z|^{2}\right) f^{\prime}(z)}{1-|f(z)|^{2}},
$$

which are defined for holomorphic functions $f: \mathbb{D} \rightarrow \mathbb{D}$ and examined in detail in [15] and [16.

Suppose $\gamma$ is a smooth curve, with a parametrization $\gamma: z=z(t), t \in[\alpha, b]$, and non-vanishing derivative. The hyperbolic curvature of $\gamma$ at the point $z=z(t)$ is given by the formula

$$
\kappa_{h}(z, \gamma)=\left(1-|z|^{2}\right) \kappa(z, \gamma)+2 \operatorname{Im}\left\{\frac{\overline{z(t)} z^{\prime}(t)}{\left|z^{\prime}(t)\right|}\right\},
$$

where $\kappa(z, \gamma)$ is the euclidean curvature of $\gamma$ at the point $z$. Let $f$ be a holomorphic and locally univalent map with $f(\mathbb{D}) \subset \mathbb{D}$. The hyperbolic curvature of $f \circ \gamma$ at a point $f(z), z=z(t) \in \gamma$, is equal to

$$
\kappa_{h}(f(z), f \circ \gamma)\left|D_{h 1} f(z)\right|=\kappa_{h}(z, \gamma)+\operatorname{Im}\left\{\frac{D_{h 2} f(z)}{D_{h 1} f(z)} \cdot \frac{z^{\prime}(t)}{\left|z^{\prime}(t)\right|}\right\} .
$$

The above formulas are presented in [16]. For the curve $C_{r}: z=r e^{i t}$, with $t \in[0,2 \pi)$, the hyperbolic geodesic curvature is equal to

$$
\kappa_{h}(z, \gamma)=\frac{1-r^{2}}{r}+2 \operatorname{Im}\left\{\frac{r e^{-i t} i r e^{i t}}{r}\right\}=\frac{1+r^{2}}{r}
$$


and its total curvature is

$$
\int_{C_{r}} \kappa_{h}\left(z, C_{r}\right) \lambda_{\mathbb{D}}(z)|d z|=\int_{0}^{2 \pi} \frac{1+r^{2}}{r} \frac{r}{1-r^{2}} d t=2 \pi \frac{1+r^{2}}{1-r^{2}}
$$

for $r \in(0,1)$. Regarding the curve $f\left(C_{r}\right)$, the hyperbolic geodesic curvature is given by (6.1). First we calculate the quantities

$$
\frac{D_{h 2} f(z)}{D_{h 1} f(z)}=\left(1-|z|^{2}\right) \frac{f^{\prime \prime}(z)}{f^{\prime}(z)}+2 \frac{1-|z|^{2}}{1-|f(z)|^{2}} \overline{f(z)} f^{\prime}(z)-2 \bar{z}
$$

and

$$
\begin{aligned}
\operatorname{Im}\left\{\frac{D_{h 2} f(z)}{D_{h 1} f(z)} \frac{i r e^{i t}}{r}\right\} & =\frac{1}{r} \operatorname{Re}\left\{\left(1-|z|^{2}\right) z \frac{f^{\prime \prime}(z)}{f^{\prime}(z)}+2 \frac{1-|z|^{2}}{1-|f(z)|^{2}} z \overline{f(z)} f^{\prime}(z)-2 \bar{z} z\right\} \\
& =\frac{1-r^{2}}{r} \operatorname{Re}\left\{z \frac{f^{\prime \prime}(z)}{f^{\prime}(z)}+2 \frac{z \overline{f(z)} f^{\prime}(z)}{1-|f(z)|^{2}}\right\}-2 r .
\end{aligned}
$$

As a result,

$$
\kappa_{h}\left(f(z), f\left(C_{r}\right)\right)\left|D_{h 1} f(z)\right|=\frac{1+r^{2}}{r}-2 r+\frac{1-r^{2}}{r} \operatorname{Re}\left\{z \frac{f^{\prime \prime}(z)}{f^{\prime}(z)}+2 \frac{z \overline{f(z)} f^{\prime}(z)}{1-|f(z)|^{2}}\right\}
$$

and with calculations we acquire

$$
\kappa_{h}\left(f(z), f\left(C_{r}\right)\right)=\frac{1-|f(z)|^{2}}{r\left|f^{\prime}(z)\right|} u(z),
$$

where $u(z)$ is defined in (1.9). Therefore, using (6.3), the hyperbolic total curvature of $f\left(C_{r}\right)$ is

$$
\begin{aligned}
\int_{f\left(C_{r}\right)} \kappa_{h}\left(w, f\left(C_{r}\right)\right) \lambda_{\mathbb{D}}(w)|d w| & =\int_{C_{r}} \kappa_{h}\left(f(z), f\left(C_{r}\right)\right) \lambda_{\mathbb{D}}(f(z))\left|f^{\prime}(z)\right||d z| \\
& =\int_{0}^{2 \pi} u\left(r e^{i t}\right) d t .
\end{aligned}
$$

Applying the Gauss-Bonnet formula (1.13) to $f(r \mathbb{D})$ viewed as a two-dimensional manifold with boundary on the Riemann surface of $f$, we obtain

$$
\int_{0}^{2 \pi} u\left(r e^{i t}\right) d t=2 \pi+4 \mathrm{~A}_{\mathrm{h}}(f(r \mathbb{D}))
$$

where $\mathrm{A}_{\mathrm{h}}(f(r \mathbb{D}))$ is the hyperbolic area of $f(r \mathbb{D})$ counting the multiplicities, since $f$ is not necessarily univalent. This implies that

$$
\int_{0}^{2 \pi} u\left(r e^{i t}\right) d t
$$

is a strictly increasing function of $r$. Furthermore, since tanh is strictly increasing, the hyperbolic total curvature is a strictly increasing function of the hyperbolic radius $\rho=\operatorname{arctanh} r$.

As a conclusion, we have the following result that leads to a better understanding and handling of the function $u(z)$.

Corollary 6.1. The function

$$
\frac{1}{2 \pi} \int_{0}^{2 \pi} u\left(r e^{i t}\right) d t
$$


is a positive and strictly increasing function of $r \in(0,1)$ for every holomorphic and locally univalent map $f: \mathbb{D} \rightarrow \mathbb{D}$.

\section{Proof of Theorem 1.5}

Recall that we have defined the function $\Phi_{h}(r)$ in (1.11). Using the calculations in Section 6, it can also be written as

$$
\Phi_{h}(r)=\frac{1-r^{2}}{2 \pi\left(1+r^{2}\right)} \int_{0}^{2 \pi}\left|u\left(r e^{i t}\right)\right| d t, \quad 0<r<1 .
$$

If the function $f$ is hyperbolically convex in $\mathbb{D}$, then

$$
\Phi_{h}(r)=\frac{1-r^{2}}{2 \pi\left(1+r^{2}\right)} \int_{0}^{2 \pi} u\left(r e^{i t}\right) d t .
$$

Applying the Gauss-Bonnet formula (1.14) to $f(r \mathbb{D})$ and using (6.4), it follows that

$$
\Phi_{h}(r)=\frac{1-r^{2}}{2 \pi\left(1+r^{2}\right)}\left(2 \pi+4 \mathrm{~A}_{\mathrm{h}}(f(r \mathbb{D}))\right)=\frac{1-r^{2}}{1+r^{2}}\left(1+\frac{2}{\pi} \mathrm{A}_{\mathrm{h}}(f(r \mathbb{D}))\right)
$$

where $\mathrm{A}_{\mathrm{h}}(f(r \mathbb{D}))$ is the hyperbolic area of $f(r \mathbb{D})$. Since $f$ is a hyperbolically convex function, it is also univalent and the hyperbolic area of $f(r \mathbb{D})$ is given by the integral

$$
\mathrm{A}_{\mathrm{h}}(f(r \mathbb{D}))=\iint_{f(r \mathbb{D})}\left(\lambda_{\mathbb{D}}(w)\right)^{2} A(d w)=\iint_{r \mathbb{D}} \frac{\left|f^{\prime}(z)\right|^{2}}{\left(1-|f(z)|^{2}\right)^{2}} A(d z),
$$

where $A$ is the area measure. We set

$$
\sigma(z)=\frac{\left|f^{\prime}(z)\right|}{1-|f(z)|^{2}}, \quad z \in \mathbb{D},
$$

which, due to the Schwarz-Pick Lemma, satisfies the inequality

$$
\sigma(z) \leq \lambda_{\mathbb{D}}(z)=\frac{1}{1-r^{2}}:=\lambda_{\mathbb{D}}(r)
$$

for $z \in C_{r}$, since the hyperbolic density is a radial function. In fact, $\sigma$ is the density of a conformal metric with cuvature -4 ; see [1, p. 12] and [16]. So,

$$
\frac{\Delta \log \sigma}{\sigma^{2}}=4>0
$$

and it follows that $\log \sigma$ is subharmonic on $\mathbb{D}$, hence $\sigma$ is subharmonic on $\mathbb{D}$. Therefore, $\sigma^{2}$ is also subharmonic on $\mathbb{D}$. Following the notation from $[25$, we denote the normalized integral mean and area integral of $\sigma^{2}$ by

and by

$$
C_{\sigma^{2}}(r)=\frac{1}{2 \pi} \int_{0}^{2 \pi} \sigma^{2}\left(r e^{i t}\right) d t
$$

$$
B_{\sigma^{2}}(r)=\frac{1}{\pi r^{2}} \iint_{r \mathbb{D}} \sigma^{2}(z) d x d y=\frac{1}{\pi r^{2}} \mathrm{~A}_{\mathrm{h}}(f(r \mathbb{D}))
$$

respectively. As a result, from (6.4),

$$
\int_{0}^{2 \pi} u\left(r e^{i t}\right) d t=2 \pi+4 \pi r^{2} B_{\sigma^{2}}(r)
$$

and $\Phi_{h}$ can also be written as

$$
\Phi_{h}(r)=\frac{1-r^{2}}{1+r^{2}}\left(1+2 r^{2} B_{\sigma^{2}}(r)\right) .
$$


For the proof of Theorem 1.5 we will need the following lemmas.

Lemma 7.1 ([16, Theorem 5]). Let $f$ be hyperbolically convex in $\mathbb{D}$. Then, for $z \in \mathbb{D}$

$$
\left|\frac{\left(1-|z|^{2}\right) f^{\prime \prime}(z)}{2 f^{\prime}(z)}+\frac{\left(1-|z|^{2}\right) \overline{f(z)} f^{\prime}(z)}{1-|f(z)|^{2}}-\bar{z}\right| \leq 1-\left(\frac{\left(1-|z|^{2}\right)\left|f^{\prime}(z)\right|}{1-|f(z)|^{2}}\right)^{2} .
$$

Lemma 7.2. Let $f$ be a hyperbolically convex function in $\mathbb{D}$. Then

$$
\left(1-r^{2}\right) C_{\sigma^{2}}(r) \leq \frac{1}{1+r}+r B_{\sigma^{2}}(r) .
$$

Proof. Since $f$ is hyperbolically convex in $\mathbb{D}$, it satisfies the inequality in Lemma 7.1. With calculations, the left-hand side part of the above inequality is greater than

$$
\frac{\left(1-|z|^{2}\right)}{2|z|}\left|u(z)-\frac{1+|z|^{2}}{1-|z|^{2}}\right|
$$

The function $u(z)>0$, for every $z \in \mathbb{D}$, due to the fact that $f$ is hyperbolically convex and using the triangle inequality at (7.5), we obtain that

$$
\frac{1+|z|^{2}}{2|z|}-\frac{1-|z|^{2}}{2|z|} u(z) \leq \frac{\left(1-|z|^{2}\right)}{2|z|}\left|u(z)-\frac{1+|z|^{2}}{1-|z|^{2}}\right| .
$$

Combining Lemma 7.1 and the inequality (7.6), we obtain

$$
\frac{1+|z|^{2}}{2|z|}-\frac{1-|z|^{2}}{2|z|} u(z) \leq 1-\left(\frac{\left(1-|z|^{2}\right)\left|f^{\prime}(z)\right|}{1-|f(z)|^{2}}\right)^{2}=1-\left(1-|z|^{2}\right)^{2} \sigma^{2}(z) .
$$

Taking the integral along the curve $C_{r}$, with respect to the hyperbolic metric, we have that

$$
\int_{C_{r}} \frac{1+r^{2}}{2 r} d s-\frac{1-r^{2}}{2 r} \int_{C_{r}} u(z) d s \leq \int_{C_{r}} d s-\left(1-r^{2}\right)^{2} \int_{C_{r}} \sigma^{2}(z) d s,
$$

which gives

$$
\pi \frac{1+r^{2}}{1-r^{2}}-\frac{1}{2} \int_{0}^{2 \pi} u\left(r e^{i t}\right) d t \leq \frac{2 \pi r}{1-r^{2}}-r\left(1-r^{2}\right) \int_{0}^{2 \pi} \sigma^{2}\left(r e^{i t}\right) d t .
$$

According to (7.3), the inequality (7.8) is written as

$\pi \frac{1+r^{2}}{1-r^{2}}-\frac{1}{2}\left(2 \pi+4 \pi r^{2} B_{\sigma^{2}}(r)\right)=\frac{2 \pi r^{2}}{1-r^{2}}-2 \pi r^{2} B_{\sigma^{2}}(r) \leq \frac{2 \pi r}{1-r^{2}}-2 \pi r\left(1-r^{2}\right) C_{\sigma^{2}}(r)$ and with calculations we have

$$
\frac{r}{1-r^{2}}-r B_{\sigma^{2}}(r) \leq \frac{1}{1-r^{2}}-\left(1-r^{2}\right) C_{\sigma^{2}}(r),
$$

which leads us to

$$
\left(1-r^{2}\right) C_{\sigma^{2}}(r) \leq \frac{1}{1+r}+r B_{\sigma^{2}}(r)
$$

for $r \in(0,1)$.

Completion of the Proof of Theorem 1.5. In order to prove that the function $\Phi_{h}$ is decreasing, we will calculate its derivative. Before that, let's note that, by 25 , Definition 2.6.7.],

$$
\frac{\mathrm{d}\left(r^{2} B_{\sigma^{2}}(r)\right)}{\mathrm{d} r}=2 r C_{\sigma^{2}}(r)
$$


and taking the derivative in (7.4),

$$
\begin{aligned}
\frac{\mathrm{d} \Phi_{h}}{\mathrm{~d} r}(r) & =-\frac{4 r}{\left(1+r^{2}\right)^{2}}\left(1+2 r^{2} B_{\sigma^{2}}(r)\right)+4 r \frac{1-r^{2}}{1+r^{2}} C_{\sigma^{2}}(r) \\
& =\frac{4 r}{\left(1+r^{2}\right)^{2}}\left(-1-2 r^{2} B_{\sigma^{2}}(r)+\left(1-r^{4}\right) C_{\sigma^{2}}(r)\right) .
\end{aligned}
$$

By Lemma 7.2 ,

$$
\left(1-r^{4}\right) C_{\sigma^{2}}(r)-2 r^{2} B_{\sigma^{2}}(r)-1 \leq \frac{1+r^{2}}{1+r}+r\left(1+r^{2}-2 r\right) B_{\sigma^{2}}(r)-1,
$$

hence (7.9) gives

$$
\frac{\left(1+r^{2}\right)^{2}}{4 r} \frac{\mathrm{d} \Phi_{h}}{\mathrm{~d} r}(r) \leq r \frac{r-1}{1+r}+r(1-r)^{2} B_{\sigma^{2}}(r)
$$

and, therefore,

$$
\frac{\left(1+r^{2}\right)^{2}}{4 r} \frac{\mathrm{d} \Phi_{h}}{\mathrm{~d} r}(r) \leq r(1-r)^{2}\left(-\lambda_{\mathbb{D}}(r)+B_{\sigma^{2}}(r)\right) .
$$

However, it holds that $B_{\sigma^{2}}(r) \leq \lambda_{\mathbb{D}}(r)$, for $r \in(0,1)$, since

$$
B_{\sigma^{2}}(r)=\frac{1}{\pi r^{2}} \mathrm{~A}_{\mathrm{h}}(f(r \mathbb{D})) \leq \frac{1}{\pi r^{2}} \mathrm{~A}_{\mathrm{h}}(r \mathbb{D})=\frac{1}{1-r^{2}}=\lambda_{\mathbb{D}}(r),
$$

using the Schwarz-Pick Lemma in (7.2) and the fact that hyperbolic area of $r \mathbb{D}$ is equal to $\frac{\pi r^{2}}{1-r^{2}}$; see [6].

The equality appears only when $\mathrm{A}_{\mathrm{h}}(f(r \mathbb{D}))=\mathrm{A}_{\mathrm{h}}(r \mathbb{D})$, for every $r \in(0,1)$, and that happens if $f$ is a conformal self-map of the unit disk. If $f$ is not a conformal self-map of $\mathbb{D}$, then $B_{\sigma^{2}}(r)<\lambda_{\mathbb{D}}(r)$, for every $r \in(0,1)$, which leads to

$$
\frac{\mathrm{d} \Phi_{h}}{\mathrm{~d} r}(r)<0
$$

due to (7.10), and $\Phi_{h}(r)$ is strictly decreasing in $(0,1)$.

Finally, if $f$ is a conformal self-map of the unit disk, due to the fact that the hyperbolic metric is invariant under conformal self-maps of $\mathbb{D}$, we have $\mathrm{A}_{\mathrm{h}}(f(r \mathbb{D}))=$ $\mathrm{A}_{\mathrm{h}}(r \mathbb{D})$. By the use of the Gauss-Bonnet formula (1.14), the curves $C_{r}$ and $f\left(C_{r}\right)$ have equal hyperbolic total curvature, hence $\Phi_{h}$ is constant and $\Phi_{h}(r) \equiv 1$ for $r \in[0,1]$.

Proof of Corollary 1.1. Combining the fact that $\Phi_{h}(r)$ is decreasing and that the limit

$$
\lim _{r \rightarrow 0} \Phi_{h}(r)=\lim _{r \rightarrow 0} \frac{1-r^{2}}{1+r^{2}}\left(1+2 r^{2} B_{\sigma^{2}}(r)\right)=1,
$$

we obtain that $\Phi_{h}(r) \leq 1$ for every $r \in(0,1)$. Hence

$$
\int_{f\left(C_{r}\right)} \kappa_{h}\left(w, f\left(C_{r}\right)\right) d s \leq \int_{C_{r}} \kappa_{h}\left(z, C_{r}\right) d s
$$

which can equivalently take the form

$$
\int_{0}^{2 \pi} u\left(r e^{i t}\right) d t \leq \frac{2 \pi\left(1+r^{2}\right)}{1-r^{2}}, \quad 0<r<1 .
$$

Equality holds, according to Theorem 1.5, if and only if $f$ is a conformal self-map of the unit disk. 


\section{ACKNOWLEDGMENT}

The author would like to thank Professor D. Betsakos for his help and advice during the preparation of this work.

\section{REFERENCES}

[1] Lars V. Ahlfors, Conformal invariants: topics in geometric function theory, McGraw-Hill Book Co., New York-Düsseldorf-Johannesburg, 1973. McGraw-Hill Series in Higher Mathematics. MR0357743

[2] Rauno Aulaskari and Huaihui Chen, Area inequality and $Q_{p}$ norm, J. Funct. Anal. 221 (2005), no. 1, 1-24. MR2124895

[3] A. F. Beardon and D. Minda, The hyperbolic metric and geometric function theory, Quasiconformal mappings and their applications, Narosa, New Delhi, 2007, pp. 9-56. MR2492498

[4] Dimitrios Betsakos, Geometric versions of Schwarz's lemma for quasiregular mappings, Proc. Amer. Math. Soc. 139 (2011), no. 4, 1397-1407. MR2748432

[5] Dimitrios Betsakos, Multi-point variations of the Schwarz lemma with diameter and width conditions, Proc. Amer. Math. Soc. 139 (2011), no. 11, 4041-4052. MR2823049

[6] Dimitrios Betsakos, Hyperbolic geometric versions of Schwarz's lemma, Conform. Geom. Dyn. 17 (2013), 119-132. MR3126908

[7] Dimitrios Betsakos and Stamatis Pouliasis, Versions of Schwarz's lemma for condenser capacity and inner radius, Canad. Math. Bull. 56 (2013), no. 2, 241-250. MR3043051

[8] Robert B. Burckel, Donald E. Marshall, David Minda, Pietro Poggi-Corradini, and Thomas J. Ransford, Area, capacity and diameter versions of Schwarz's lemma, Conform. Geom. Dyn. 12 (2008), 133-152. MR2434356

[9] Peter L. Duren, Univalent functions, Grundlehren der Mathematischen Wissenschaften [Fundamental Principles of Mathematical Sciences], vol. 259, Springer-Verlag, New York, 1983. MR708494

[10] Michael E. Gage, An isoperimetric inequality with applications to curve shortening, Duke Math. J. 50 (1983), no. 4, 1225-1229. MR726325

[11] G. H. Hardy, J. E. Littlewood, and G. Pólya, Inequalities, Cambridge Mathematical Library, Cambridge University Press, Cambridge, 1988. Reprint of the 1952 edition. MR944909

[12] W. K. Hayman, Multivalent functions, 2nd ed., Cambridge Tracts in Mathematics, vol. 110, Cambridge University Press, Cambridge, 1994. MR 1310776

[13] Maurice Heins, Selected topics in the classical theory of functions of a complex variable, Athena Series: Selected Topics in Mathematics, Holt, Rinehart and Winston, New York, 1962. MR0162913

[14] Antoine Henrot and Othmane Mounjid, Elasticae and inradius, Arch. Math. (Basel) 108 (2017), no. 2, 181-196. MR 3605064

[15] William Ma, David Minda, and Diego Mejia, Hyperbolically 1-convex functions, Ann. Polon. Math. 84 (2004), no. 3, 185-202. MR.2110925

[16] Wan Cang Ma and David Minda, Hyperbolically convex functions, Ann. Polon. Math. 60 (1994), no. 1, 81-100. MR1295110

[17] Wan Cang Ma and David Minda, Geometric properties of hyperbolic geodesics, Quasiconformal mappings and their applications, Narosa, New Delhi, 2007, pp. 165-187. MR2492503

[18] Diego Mejía and David Minda, Hyperbolic geometry in k-convex regions, Pacific J. Math. 141 (1990), no. 2, 333-354. MR.1035447

[19] David Minda, Applications of hyperbolic convexity to Euclidean and spherical convexity, J. Analyse Math. 49 (1987), 90-105. MR928508

[20] M. Papadimitrakis, On convexity of level curves of harmonic functions in the hyperbolic plane, Proc. Amer. Math. Soc. 114 (1992), no. 3, 695-698. MR.1086339

[21] John A. Pfaltzgraff and Bernard Pinchuk, A variational method for classes of meromorphic functions, J. Analyse Math. 24 (1971), 101-150. MR0281899

[22] George Pólya and Gabor Szegö, Problems and theorems in analysis. II, Classics in Mathematics, Springer-Verlag, Berlin, 1998. Theory of functions, zeros, polynomials, determinants, number theory, geometry; Translated from the German by C. E. Billigheimer; Reprint of the 1976 English translation. MR 1492448 
[23] Christian Pommerenke, Univalent functions, Vandenhoeck \& Ruprecht, Göttingen, 1975. With a chapter on quadratic differentials by Gerd Jensen; Studia Mathematica/Mathematische Lehrbücher, Band XXV. MR0507768

[24] T. Radó, Subharmonic Functions, Springer, 1937.

[25] Thomas Ransford, Potential theory in the complex plane, London Mathematical Society Student Texts, vol. 28, Cambridge University Press, Cambridge, 1995. MR1334766

[26] Michael Spivak, A comprehensive introduction to differential geometry. Vol. V, 2nd ed., Publish or Perish, Inc., Wilmington, Del., 1979. MR.532834

[27] E. Study, Vorlesungen über ausgewählte Gegenstände der Geometrie (German edition), Cornell University Library, 1911.

Department of Mathematics, Aristotle University of Thessaloniki, 54124, ThessaLONIKI, GREECE

Email address: mkouroue@math.auth.gr 